ARTICLE

Received 22 Mar 2015 | Accepted 12 Sep 2015 | Published 16 Oct 2015

DOI: $10.1038 /$ ncomms9622 OPEN

\title{
Encapsulation of sulfur with thin-layered nickel-based hydroxides for long-cyclic lithium-sulfur cells
}

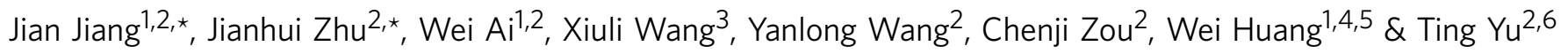

Elemental sulfur cathodes for lithium/sulfur cells are still in the stage of intensive research due to their unsatisfactory capacity retention and cyclability. The undesired capacity degradation upon cycling originates from gradual diffusion of lithium polysulfides out of the cathode region. To prevent losses of certain intermediate soluble species and extend lifespan of cells, the effective encapsulation of sulfur plays a critical role. Here we report an applicable way, by using thin-layered nickel-based hydroxide as a feasible and effective encapsulation material. In addition to being a durable physical barrier, such hydroxide thin films can irreversibly react with lithium to generate protective layers that combine good ionic permeability and abundant functional polar/hydrophilic groups, leading to drastic improvements in cell behaviours (almost 100\% coulombic efficiency and negligible capacity decay within total 500 cycles). Our present encapsulation strategy and understanding of hydroxide working mechanisms may advance progress on the development of lithium/sulfur cells for practical use.

\footnotetext{
${ }^{1}$ Nanyang Technological University-Nanjing Tech Center of Research and Development, Nanjing Tech University, Nanjing 211816, China. ${ }^{2}$ Division of Physics and Applied Physics, School of Physical and Mathematical Sciences, Nanyang Technological University, Singapore 637371, Singapore. ${ }^{3}$ State Key Laboratory of Silicon Materials, Key Laboratory of Advanced Materials and Applications for Batteries of Zhejiang Province, and School of Materials Science and Engineering, Zhejiang University, Hangzhou 310027, China. ${ }^{4}$ Key Laboratory of Flexible Electronics (KLOFE), Institute of Advanced Materials (IAM), Nanjing Tech University (NanjingTech), 30 South Puzhu Road, Nanjing 211816, Jiangsu, China. ${ }^{5}$ Key Laboratory for Organic Electronics and Information Displays and Institute of Advanced Materials (IAM), Nanjing University of Posts \& Telecommunications, 9 Wenyuan Road, Nanjing 210023, Jiangsu, China. ${ }^{6}$ Department of Physics, Faculty of Science, National University of Singapore, Singapore 117542, Singapore. ${ }^{\star}$ These authors contributed equally to this work. Correspondence and requests for materials should be addressed to T.Y. (email: yuting@ntu.edu.sg) or to W.H. (email: iamwhuang@njtech.edu.cn).
} 
ithium/sulfur ( $\mathrm{Li} / \mathrm{S})$ cells are promising energy storage devices to power electric vehicles for long-distance driving (>300 miles per charge) due to their upper theoretical energy density and lower price in comparison with currently used Li-ion cells ${ }^{1-3}$. According to charge/discharge voltage profiles or electrolytes applied in Li/S cell systems, the cathode materials can be generally categorized into two types: (1) the elemental sulfur (aggregated cyclo-octasulfur $\mathrm{S}_{8}$ ) and (2) a series of sulfur-derived composites ${ }^{4}$. Elemental $\mathrm{S}_{8}$ owns overwhelming advantages over the synthetic thionic composites. On one hand, it is environmentally benign and abundant in nature, hence readily available and fairly cheap in markets; on the other hand, when coupled with $\mathrm{Li}$ metal anode, it operates at a safer voltage of $\sim 2.15 \mathrm{~V}$ (versus $\mathrm{Li} / \mathrm{Li}^{+}$) compared with conventional Li-insertion compounds $\left(\sim 3-4.5 \mathrm{~V}\right.$ versus $\left.\mathrm{Li} / \mathrm{Li}^{+}\right)$, and offers a higher energy density than thionic counterparts ${ }^{4-7}$. The $\mathrm{S}_{8}$ can exhibit a total theoretical capacity of $1,672 \mathrm{mAh} \mathrm{g}^{-1}$ when undergoing an overall redox reaction of $\mathrm{S}_{8}+16 \mathrm{Li}^{+}+16 \mathrm{e}^{-} \leftrightarrow 8 \mathrm{Li}_{2} \mathrm{~S}$ (ref. 8). The corresponding energy density reaches as high as $\sim 2,567 \mathrm{Wh} \mathrm{kg}^{-1}$, more than sixfold that of commercial $\mathrm{LiCoO}_{2} / \mathrm{C}$ cells $\left(\sim 387 \mathrm{Wh} \mathrm{kg}^{-1}\right)^{9}$.

The development of $\mathrm{Li} / \mathrm{S}$ cells based on pure $\mathrm{S}_{8}$ cathode, however, is impeded by several challenges unfortunately. Primarily, both $\mathrm{S}_{8}$ and the discharged end products $\mathrm{Li}_{2} \mathrm{~S}_{2} / \mathrm{Li}_{2} \mathrm{~S}$ are insulators ${ }^{10}$. Particularly noteworthy is that, $\mathrm{Li}_{2} \mathrm{~S}$ is an extremely poor electrically/ionically conducting material with electrical conductivity of $\sim 10^{-30} \mathrm{~S} \mathrm{~cm}^{-1}$ and $\mathrm{Li}^{+}$diffusivity of $\sim 10^{-15} \mathrm{~cm}^{2} \mathrm{~s}^{-1}$, which inevitably poses inferior cell kinetics on charge transfer and low utilization efficiency of $\mathrm{S}_{8}$ (ref. 3). Next is the undesired self-discharge issue in $\mathrm{Li} / \mathrm{S}$ cells. Unlike $\mathrm{LiCoO}_{2} / \mathrm{C}$ cells with stable passivation layers covering on electrode interfacial surfaces, in $\mathrm{Li} / \mathrm{S}$ cell system, the $\mathrm{S}_{8}$ cathode exposed in electrolyte under a fully charged state tends to react with $\mathrm{Li}^{+}$ gradually, convert to polysulfide species and dissolve into the electrolyte, which eventually results in a static energy decrease in cell capacity ${ }^{11}$. Last but the foremost, along repeated charge/ discharge procedures, the unavoidable dissolution and loss of intermediate polysulfides $\left(\mathrm{Li}_{2} \mathrm{~S}_{\mathrm{n}}, n=3-8\right)$, together with their notable 'shuttle effects' between the anode and cathode, will inevitably lead to the formidable issues of severe capacity decay, low coulombic efficiency and limited cyclic life $\mathrm{e}^{12-14}$.

The long-lasting and stable cyclic behaviour is undoubtedly the fundamental prerequisite for future commercialization of $\mathrm{Li} / \mathrm{S}$ cells. To prolong the cyclic lifetime, a worldwide-recognized approach is to wrap the active $S_{8}$ with an effective physical barrier so as to solidly confine the soluble intermediate polysulfides in the cathode region ${ }^{3,15-27}$. Several $S_{8}$ encapsulation avenues have been presented to date, normally by the use of carbon materials (typically like two-dimensional graphene), conductive polymers or diverse inorganic coating layers ${ }^{15-27}$. Though using intrinsically mesoporous carbons is taken for granted as an ideal strategy to restrict polysulfide molecules in cathode matrices $^{15-19}$, actually weak interactions between non-polar carbon materials and $\mathrm{Li}_{2} \mathrm{~S}_{\mathrm{n}}$ reduce the ability to bind and entrap these soluble polar species ${ }^{20}$. As a consequence, only use of carbon materials can benefit the cell kinetics/reversibility and alleviate the capacity fading but still fail to resolve the short cyclic problem. Though soft conductive polymers or organic frameworks are also conceived as preferred and competitive inhibitors to hinder the polysulfides shuttling, their behaviours on elongating the cell lifetime are yet far from perfect $^{21-24}$. By contrast, encapsulations realized via inorganic species (for example, $\mathrm{S}_{8}-\mathrm{TiO}_{2}$ yolk-shell nanoarchitectures) hold great potential to build long-term cyclic Li/S cells ${ }^{25-27}$. Nevertheless, such state-of-the-art core-shell nanohybrids yet show little promise in broad commercial use owing to limitations such as low tap density and high surface areas of nanomaterials ${ }^{28}$, excess interparticle boundaries contained in electrode systems ${ }^{29}$ and so on. Therefore, to overcome the major cyclic constraint and bring $\mathrm{Li} / \mathrm{S}$ cells a step closer to commercialization, seeking for reliable 'armors' fit for $\mathrm{S}_{8}$ cathode and an efficient encapsulating strategy applicable to bulks ( $\sim 20 \mu \mathrm{m}$ scale) for higher volumetric energy is urgently pursued.

Herein, we demonstrate the feasibility of adopting semiconducting nickel nitrate hydroxide $\left(\mathrm{Ni}_{3}\left(\mathrm{NO}_{3}\right)_{2}(\mathrm{OH})_{4}\right.$, denoted as $\mathrm{NNH}$ ), one type of thin-layered $\alpha-\mathrm{Ni}(\mathrm{OH})_{2}$ often applied in nickel-metal hydride cells and supercapacitors, as a novel and effective encapsulation material for $\mathrm{S}_{8}$ cathode. More than a durable physical shell, NNH is capable to irreversibly react with $\mathrm{Li}^{+}$in initial tens of cycles, turning into reliable $(\mathrm{Li}, \mathrm{Ni})$-mixed hydroxide compounds with a combination of good $\mathrm{Li}^{+}$ permeability/accessibility and copious functional polar/hydrophilic groups (for example, hydrophilic groups, surface hydroxyl groups and so on) existing in cathode systems ${ }^{12,27}$. In this work, we choose the simple hybrid of $\mathrm{S}_{8} @$ carbon black $\left(\mathrm{S}_{8} @ \mathrm{CB}\right.$; with a central dimension of $\sim 20 \mu \mathrm{m}$ ), as a paradigm to investigate the electrochemical functions of NNH. By using such a smart coreshell $\mathrm{S}_{8} @ \mathrm{CB} @ N N H$ hybrid as the cathode, we eventually achieve drastic improvements in capacity retention (almost $\sim 500 \%$ capacity rise when compared with the case of bare $\mathrm{S}_{8} @ \mathrm{CB}$ ) and long-term cyclic stability (negligible capacity decay within total 500 cycles). Our present work may open up a feasible and effective concept of using thin-layered transition metal hydroxides as a promising class of encapsulation materials to build better Li/S cells.

\section{Results}

Synthesis and characterization. The entire fabrication of coreshell $\mathrm{S}_{8} @ \mathrm{CB} @ N N H$ bulky hybrids was schematically shown in Fig. 1a (see Methods section for details; basic characterizations on $\mathrm{NNH}$ are involved in Supplementary Fig. 1). In brief, the preparation of $\mathrm{S}_{8} @ \mathrm{CB}$ particles was achieved by impregnation of molten $\mathrm{S}_{8}$ into $\mathrm{CB}$ matrices. The choice of $\mathrm{CB}$ powder, which has been long commercialized in cell technologies, as the $S_{8}$ carrier is due to its large surface-to-volume ratios, excellent electrically conducting properties and far lower cost than other counterparts like advanced mesoporous carbons, graphene and carbon nanotubes and so on. Another notable reason is that there are numerous nanosized cavities (Fig. 1b,c) distributed in between $\mathrm{CB}$ nanoparticles, as reflected by the pore-size distribution plot (Supplementary Fig. 2). These interspaces (randomly distributed in a wide range of $\sim 1-50 \mathrm{~nm}$ ) can not only supply abundant 'reservoir' places to accommodate $S_{8}$ but also help to downsize the $\mathrm{S}_{8}$ bulks into nanoparticles for preferable cell kinetics. Figure 1d,e shows typical scanning electron microscopy (SEM) observations on $\mathrm{S}_{8} @ \mathrm{CB}$ powders. During the melting treatment, the $S_{8}$ powders have definitely fused into a liquid state, penetrated/embedded into certain cavities and merged together with CB-conducting agents. The size for entire bulks is centred at $\sim 20 \mu \mathrm{m}$ (Supplementary Fig. 3), while the diameter of individual $\mathrm{S}_{8} @ \mathrm{CB}$ subunit lies in a range of $\sim 20-100 \mathrm{~nm}$. The following procedure was undertaken at a low temperature of $95^{\circ} \mathrm{C}$ in an aqueous solution where the $\mathrm{S}_{8} @ \mathrm{CB}$ hybrids are uniformly packaged by $\mathrm{NNH}$. The reaction of $\mathrm{Ni}$ salts with ammonium $\left(\mathrm{NH}_{4} \mathrm{OH}\right)$ yielded from the progressive hydrolysis of hexamethylenetetramine $\quad\left(\mathrm{C}_{6} \mathrm{H}_{12} \mathrm{~N}_{4}+10 \mathrm{H}_{2} \mathrm{O} \rightarrow 6 \mathrm{HCHO}+4 \mathrm{NH}_{4} \mathrm{OH}\right)$ guarantees an intact encapsulation of NNH layers on each $\mathrm{S}_{8} @ \mathrm{CB}$ particle. Fresh $\mathrm{S}_{8} @ \mathrm{CB} @ N N H$ products are then washed by centrifugation and dried at $60^{\circ} \mathrm{C}$ in an electronic oven. S.e.m. observations (Fig. 1f,g) reveal that the as-formed $\mathrm{S}_{8} @ \mathrm{CB} @ N N H$ hybrids possess a well-defined core-shell configuration; all of the 

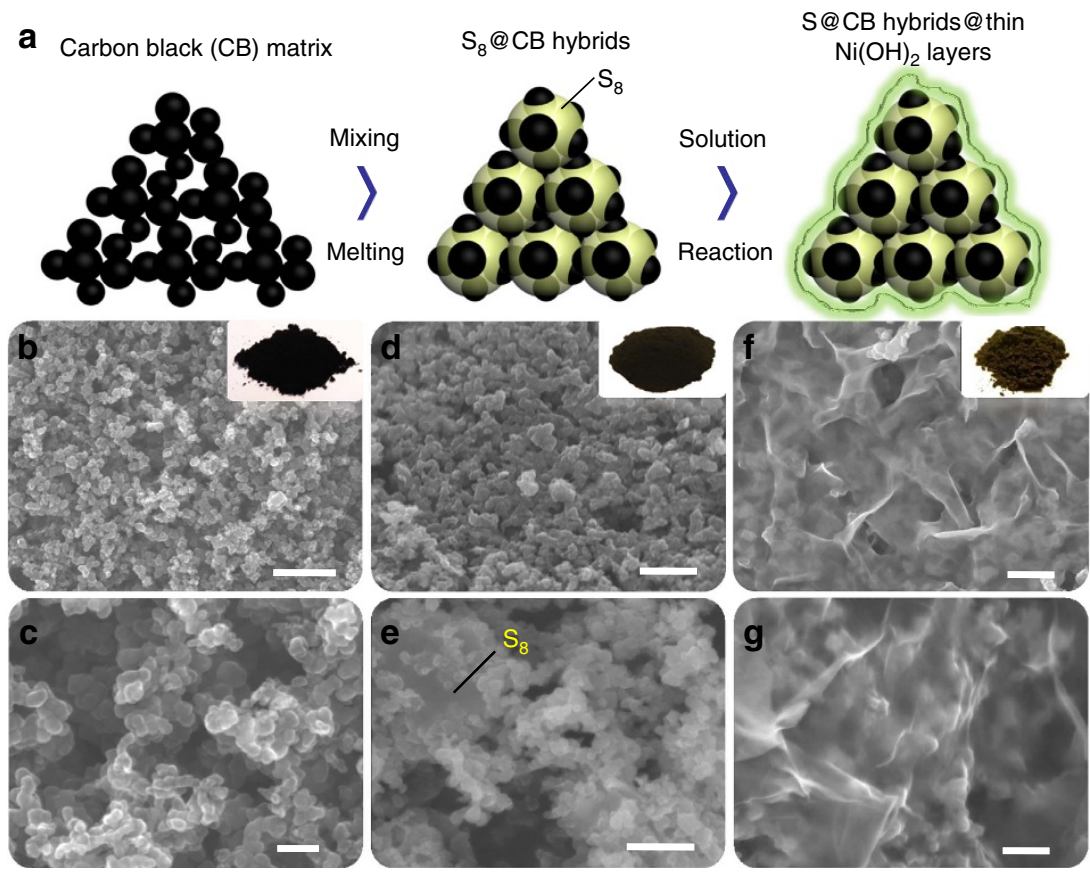

Figure 1 | Schematic and s.e.m. images showing the fabrication of $\mathbf{S}_{\mathbf{8}} @ \mathbf{C B} @ \mathbf{N N H}$ hybrids. (a) A general schematic for the entire fabrication procedures. Basic s.e.m. observations towards $(\mathbf{b}, \mathbf{c})$ CB powders, $(\mathbf{d}, \mathbf{e}) \mathrm{S}_{8} @$ CB intermediates and $(\mathbf{f}, \mathbf{g}) \mathrm{S}_{8} @ \mathrm{CB} @ N N H$ hybrids. The inset pictures correspond to their optical images, respectively. Scale bars, $200 \mathrm{~nm}(\mathbf{b}, \mathbf{d}), 50 \mathrm{~nm}(\mathbf{c}, \mathbf{g})$ and $100 \mathrm{~nm}(\mathbf{e}, \mathbf{f})$.

nanosized $\mathrm{S}_{8} @ \mathrm{CB}$ subunits have been intimately packaged within the layered film structures. Also, the stepwise evolutions of $\mathrm{S}_{8} @ \mathrm{CB} @ \mathrm{NNH}$ are further confirmed in parallel using energy dispersive X-ray spectroscopy (EDX) to monitor the entire fabrication flow (see details in Supplementary Fig. 4).

A special focus is then put on the ultimate products of $\mathrm{S}_{8} @ \mathrm{CB} @ N N H$. Figure 2a-d shows their basic s.e.m. observations. The large-area SEM detection (Fig. 2a) illustrates that the selfassembled $\mathrm{S}_{8} @ \mathrm{CB} @ \mathrm{NNH}$ product owns a bulky structural feature ( $\sim 20 \mu \mathrm{m}$ upward in width) and an interesting heart-shaped geometric profile. No naked areas are observed on bulky $\mathrm{S}_{8} @ \mathrm{CB} @ \mathrm{NNH}$ hybrids, ascertaining our success in perfectly packaging $\mathrm{S}_{8} @ \mathrm{CB}$ into $\mathrm{NNH}$ shells. Close-up s.e.m. images (Fig. 2b,c) furthermore uncover their detailed structural information. Definitely, every $\mathrm{S}_{8} @ \mathrm{CB}$ nanoparticle unit has been overall and closely wrapped with NNH 'armors', as also confirmed by transmission electron microscope (TEM) image in Supplementary Fig. 5. Note that our used encapsulating material is supple enough to conform well to arbitrary body shapes of introduced precursors, enabling a tight and intact encapsulation of involved $\mathrm{S}_{8} @ \mathrm{CB}$ powders. Moreover, the $\mathrm{NNH}$ films are extremely thin (the thickness for one NNH layer is only $\sim 7 \mathrm{~nm}$, as evidenced by pioneering works) ${ }^{30,31}$; TEM and s.e.m. observations (Supplementary Figs 1e,f and 6) further reveal the distribution of pore defects on NNH surface. Such a thin-film characteristic, together with intrinsic porosity properties (for example, micropores/mesopores on $\mathrm{NNH}$ or in between these overlaid structures) may allow $\mathrm{Li}^{+}$to move readily across the outer protective layers, pledging the expedite ionic access/ permeation to inner places of entire cathode. Figure $2 \mathrm{e}-\mathrm{h}$ displays the EDX elemental mapping records towards one bulky $\mathrm{S}_{8} @ \mathrm{CB} @ N N H$ product (see its SEM morphology in Fig. 2d). The mapping images visually declare a homogeneous dispersion of $\mathrm{C}$, $\mathrm{S}, \mathrm{Ni}$ and $\mathrm{O}$ atoms in this hybrid. In addition, we purposely carry out a line-scan analysis on a selected section of $\mathrm{S}_{8} @ \mathrm{CB} @ \mathrm{NNH}$ (Fig. 2i). Though there is a visible thick layer of $\mathrm{NNH}$ covering this region, the involved $S$ element still takes up the largest proportion (rather than $\mathrm{Ni}$ or $\mathrm{O}$ element) and stays evenly distributed. To clarify the actual contents of $\mathrm{C}, \mathrm{NNH}$ and $\mathrm{S}_{8}$ in $\mathrm{S}_{8} @ \mathrm{CB} @ \mathrm{NNH}$ hybrids, the powder samples thus undergo an acid immersion treatment followed by a thermogravimetric (TG) measurement (see experimental details in Methods section). The stepwise weight losses recorded in Fig. 2j reflect the detailed compositions of $\mathrm{S}_{8} @ \mathrm{CB} @ \mathrm{NNH}$, containing $~ 8.2 \%$ of $\mathrm{NNH}$, $\sim 13.4 \%$ of $\mathrm{CB}$ and $\sim 78.4 \%$ of active $\mathrm{S}_{8}$. Figure $2 \mathrm{k}$ shows the X-ray powder diffraction (XRD) pattern of $\mathrm{S}_{8} @ \mathrm{CB} @ N N H$. In addition to distinguishable signals from $\mathrm{NNH}$ and amorphous $\mathrm{CB}$, all other strong diffraction peaks stem from the crystalline $\mathrm{S}_{8}$ (JCPDS no. 08-0247).

Electrochemical performance. To evaluate the electrochemical properties of $\mathrm{S}_{8} @ \mathrm{CB} @ \mathrm{NNH}$ hybrids, the as-synthesized electrodes are initially subjected to a long-term cyclic test in a potential window of $\sim 1.5-3 \mathrm{~V}$ at a constant current rate of $0.2 \mathrm{C}$ (335 $\mathrm{mAg}^{-1} ; 1 \mathrm{C}=1,672 \mathrm{mAg}^{-1}$ ). To verify and highlight the cooperative functions of $\mathrm{NNH}$, bare $\mathrm{S}_{8} @ \mathrm{CB}$ cathodes are also measured underneath the same conditions for comparison (Fig. 3a). The $\mathrm{S}_{8} @ \mathrm{CB}$ cathode, as a whole, exhibits poor electrochemical behaviours including rapid decay in both specific capacity and coulombic efficiency and short cyclic life period. The discharge capacity on the first cycle achieves $1,345 \mathrm{mAh}^{-1}$ but it declines ceaselessly to a low capacitive value (less than $\sim 200 \mathrm{mAhg}^{-1}$ after 300 cycles). On cycling, an increasing divergence between the charge and discharge capacity highly suggests the severe degradation on the coulombic efficiency. At the 300th cycle, only a coulombic efficiency value of $\sim 52 \%$ is sustained. The drastic decrease in capacity/coulombic efficiency would be mainly attributed to formidable kinetic issues in $\mathrm{Li} / \mathrm{S}$ cell systems. A fraction of yielded polysulfide molecules that should be trapped in carbon reservoirs gradually migrate out of the cathode, and they may not be reversibly used again ${ }^{32}$. Even worse, undesired side reactions between these highly reactive polysulfide anions and electrolyte solvents would furthermore 

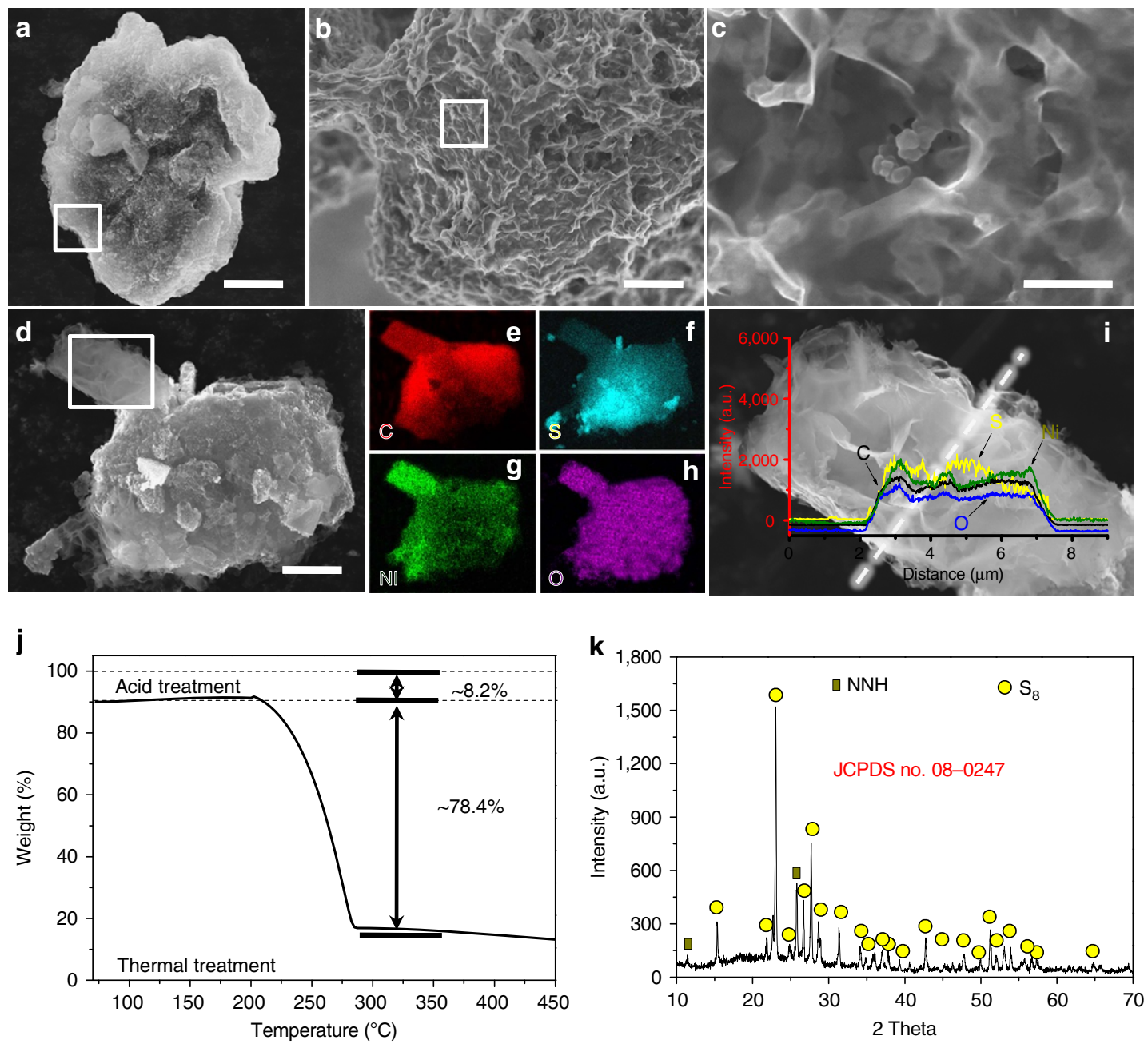

Figure 2 | Characterizations of the $\mathbf{S}_{\mathbf{8}} @ \mathbf{C B} @$ NNH bulky hybrids. (a-d) S.e.m. images of $\mathrm{S}_{8} @ C B @ N N H$ hybrids. (e-h) EDX elemental mapping records towards one typical $\mathrm{S}_{8} @ \mathrm{CB} @ N N H$ bulky sample, and (i) a line-scan analysis on a selected section of this hybrid product. (j) A plot recording weight losses of $\mathrm{S}_{8} @ \mathrm{CB} @ N N H$ products during an acid immersion treatment combined with a TG measurement. (k) XRD pattern of synthesized $\mathrm{S}_{8} @ C B @ N N H$ powders. Scale bars, $10 \mu \mathrm{m}(\mathbf{a}, \mathbf{d}), 1 \mu \mathrm{m}(\mathbf{b})$ and $100 \mathrm{~nm}(\mathbf{c})$.

speed up the capacity losses ${ }^{33}$. By sharp contrast, the $\mathrm{S}_{8} @ \mathrm{CB} @ \mathrm{NNH}$ cathode shows far better charge/discharge performance over 500 cycles. Among initial few cycles, the capacity of $\mathrm{S}_{8} @ \mathrm{CB} @ \mathrm{NNH}$ in the beginning drops from the initial $\sim 968 \mathrm{mAhg}^{-1}$ to a bottom level of $\sim 786 \mathrm{mAhg}^{-1}$ (ninth cycle), which is mostly due to a delayed electrolyte infiltration into a well-capsulated structure. In subsequent 100 cycles (from the 50th to 150th cycle), the output for $\mathrm{S}_{8} @ \mathrm{CB} @ \mathrm{NNH}$-based cells rises progressively (since more $S_{8}$ becomes activated) until an electrochemical equilibrium state is built. About 150 cycles later, the capacity grows slowly to a maximum reversible value of $\sim 1,326 \mathrm{mAh} \mathrm{g}^{-1}$ (410th cycle) and maintains stabilized at $\sim 1,250 \mathrm{mAh} \mathrm{g}^{-1}$ to the end of cycling (no similar capacity-rise phenomena happen on $\mathrm{S}_{8} @ \mathrm{CB}$ cathodes). Capacitive growth in this stage may be attributed to unavoidable structural fatigue/ damage of outer layers because film breaking may provide new open-up places and accordingly more available routes for $\mathrm{Li}^{+}$to reach inner deep regions wherein the $\mathrm{S}_{8}$ is deadly trapped. Till the 300th cycle, the $\mathrm{S}_{8} @ \mathrm{CB} @ \mathrm{NNH}$ cathode still stably outputs a capacity of $\sim 1,164 \mathrm{mAh}^{-1}$, almost six times greater than that of $S_{8} @ C B$. Also notice that unlike $S_{8} @ C B$-based cells, there is actually no capacity fading for $\mathrm{S}_{8} @ \mathrm{CB} @ N N H$ cathodes within prime 410 cycles. The coulombic efficiency always stays beyond $\sim 98 \%$, indicative of outstanding electrochemical reversibility of
$\mathrm{S}_{8} @ \mathrm{CB} @ N N H$ cathodes. Assuming the utilized $\mathrm{S}_{8}$ is fully activated and contributes a theoretical capacity, the utilization ratio of active $\mathrm{S}_{8}$ (calculated based on the maximum capacity in cycling) reaches up to $\sim 78.3 \%$, with a substantial rise by $59.5 \%$ in contrast with that in $\mathrm{S}_{8} @ \mathrm{CB}$ case $(49.1 \%)$. Above evidences fully confirm that our facile encapsulation of $\mathrm{NNH}$ indeed plays a positive role in inhibiting the outward diffusion of long-chain soluble polysulfides from the cathode, and thus greatly promoting the long-cyclic cell performance.

With the goal of attaining valuable insights into the cyclic information, we carefully analyze the charge/discharge voltage profiles of $\mathrm{S}_{8} @ \mathrm{CB} @ N N H$ cathode. For better comparisons between the pristine $\mathrm{S}_{8} @ \mathrm{CB}$ and final $\mathrm{S}_{8} @ \mathrm{CB} @ \mathrm{NNH}$ hybrid on cell performances, additional charge/discharge profiles of $\mathrm{S}_{8} @ \mathrm{CB}$ have also been provided (Supplementary Fig. 7). Figure 3b evidently shows that in the first electrochemical process, only a distorted discharge curve (rather than the smooth and distinct plateaus of bare $\mathrm{S}_{8} @ \mathrm{CB}$ ) is recorded. However, the discharge profiles lying at the 50th and subsequent cycles change a lot. Two main plateaus are present obviously, corresponding to the reduction of $\mathrm{S}_{8}$ into complex solubilized polysulfides (for example, $\mathrm{Li}_{2} \mathrm{~S}_{8}, \mathrm{Li}_{2} \mathrm{~S}_{6}, \mathrm{Li}_{2} \mathrm{~S}_{4}$ and so on) over a potential range of $\sim 2.1-2.3 \mathrm{~V}$ and ultimate formation of solid-state $\mathrm{Li}_{2} \mathrm{~S}_{2} / \mathrm{Li}_{2} \mathrm{~S}$ at $\sim 2.0 \mathrm{~V}$. Plateaus in later charge profiles are related to converse 
a

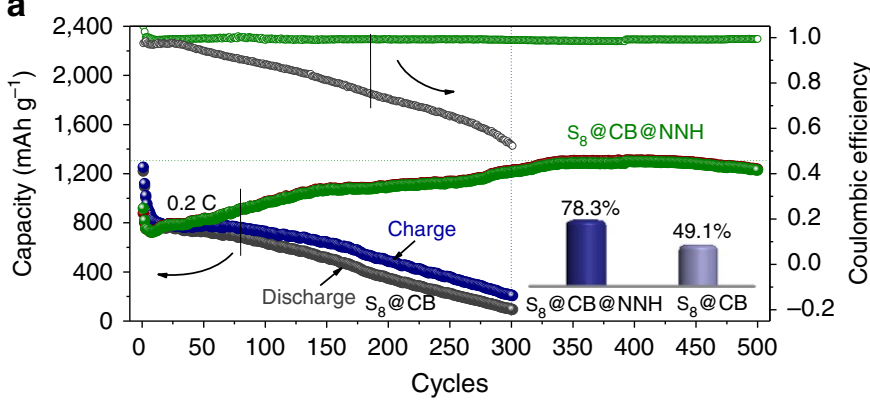

C

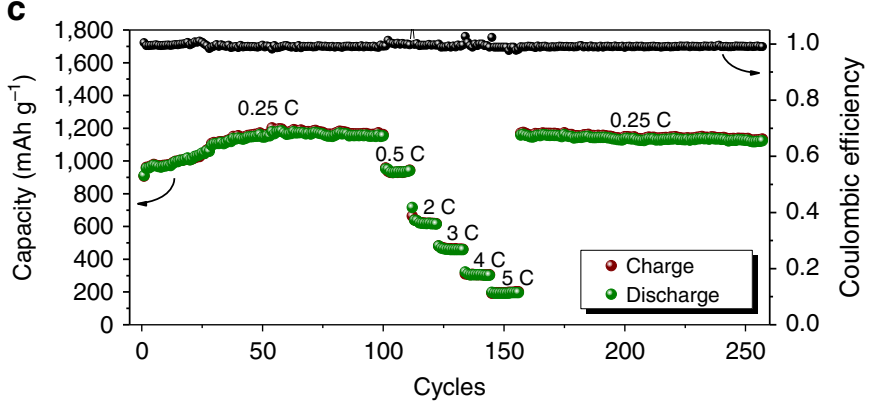

b

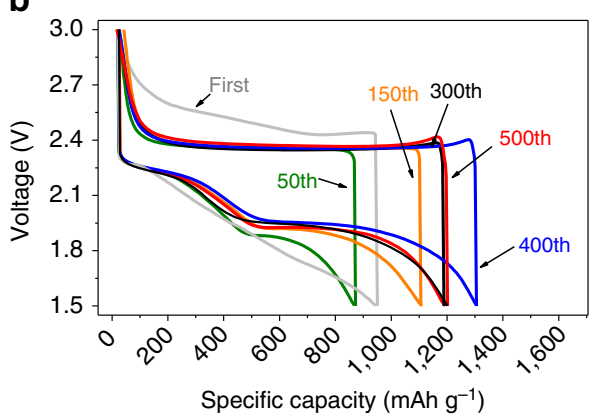

d

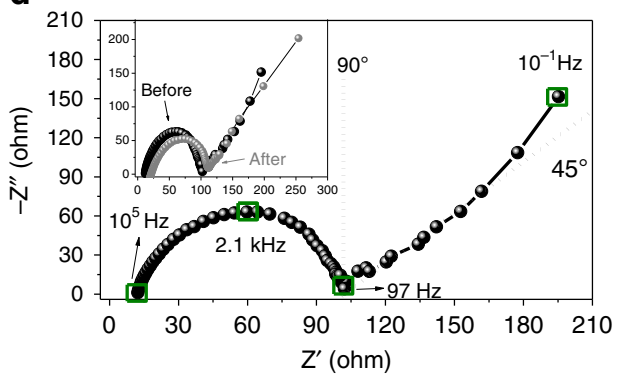

Figure 3 | Cell performance of the $\mathbf{S}_{\mathbf{8}} @ \mathbf{C B} @ N$ NH hybrids. (a) Long-term cyclic testing of $\mathrm{S}_{8} @ C B @ N N H$ and $\mathrm{S}_{8} @ C B$ at a current rate of $0.2 \mathrm{C}$ in a potential window of $\sim 1.5-3 \mathrm{~V}$. The inset shows their utilization ratio of active $S_{8}$. (b) Charge/discharge voltage profiles, (c) programmed cyclic responses and (d) electrochemical impedance spectrum of $\mathrm{S}_{8} @ \mathrm{CB} @ N N H$ cathode.

reactions from lithium sulfides to metastable polysulfides, and finally backward to the primitive $S_{8}$. The appealing capacity-rise phenomenon is also recognized. The discharge plateau nearby $\sim 2.0 \mathrm{~V}$ extended gradually along with increased cyclic numbers, signifies that certain enhanced capacities are tightly correlated with complex conversions between highly ordered polysulfides and short-chain $\mathrm{Li}_{2} \mathrm{~S}_{2} / \mathrm{Li}_{2} \mathrm{~S}$. We consider that the inner deep dispersions of active $S_{8}$ and postponed electrolyte penetration led by outer enclosure of NNH multi-layers may well account for the presence of this unique capacitive activation. Apart from that, frequent $\mathrm{Li}^{+}$migrations would endow exterior NNH layers with tiny micropore/mesopore architectures, quite favourable for $\mathrm{Li}^{+}$ diffusion; as a consequence, better utilization of $S_{8}$ actives and easier conversion reactions of $\mathrm{Li}_{2} \mathrm{~S}_{4} / \mathrm{Li}_{2} \mathrm{~S}$ can be observed due to progressively enhanced electrochemical kinetics. Rate capabilities are further estimated by a cyclic test under programmed current densities (Fig. 3c). Corresponding charge/discharge voltage profiles at varied current rates are also displayed in Supplementary Fig. 8. The cell exhibits a stable cyclic behaviour at each current speed, with all coulombic efficiencies nearly approaching $\sim 100 \%$. Allowing for sufficient electrode activation, the $\mathrm{S}_{8} @ \mathrm{CB} @ \mathrm{NNH}$ cathode preliminarily suffers from continuous 100 cycles under a constant current rate of $0.25 \mathrm{C}$, with a delivered capacity of $\sim 1,153 \mathrm{mAhg}^{-1}$ (at the 100th cycle). Afterwards, the cell runs sequentially at $0.5,2,3,4$ and $5 \mathrm{C}$, enabling an output discharge capacity of $\sim 943, \sim 610, \sim 467$, $\sim 289$ and $\sim 195 \mathrm{mAhg}^{-1}$, respectively. This electrode rate performance is much superior to those in previous examples having similar ' $\mathrm{S}_{8}$-in-carbon matrix' configurations ${ }^{34-36}$. Even when the current rate suddenly switches back to $0.25 \mathrm{C}$, an exceptional specific capacity of $1,149 \mathrm{mAhg}^{-1}$ is able to be retained (almost $\sim 100 \%$ recovery ratio in stored capacity). Figure $3 \mathrm{~d}$ presents the electrochemical impedance spectrum of assembled $\mathrm{S}_{8} @ \mathrm{CB} @ \mathrm{NNH}$ cells at an open-circuit voltage. The hybrid electrode of $\mathrm{S}_{8} @ \mathrm{CB} @ \mathrm{NNH}$, albeit with an exterior package of $\mathrm{NNH}$ films, shows a comparable semicircle diameter to bare $\mathrm{S}_{8} @ \mathrm{CB}$ cathodes among the high-frequency region
(Supplementary Fig. 9). There are no considerable differences in terms of charge-transfer resistance $\left(R_{c t}\right)$, representing that such incorporation of $\mathrm{NNH}$ thin layers would not have a great effect on charge transfer. Moreover, little change is observed on $R_{c t}$ impedance (see the inset in Fig. 3d) between original cells and the ones after 400 fatigue cycles, once again ensuring the good electrochemical stability of $\mathrm{S}_{8} @ \mathrm{CB} @ N N H$ cathode.

Working principles of $\mathrm{NNH}$ for prolonged $\mathrm{Li} / \mathrm{S}$ cells. To make certain the change on discharge voltage profiles aforementioned, a cyclic voltammetry (CV) test at a slow scan rate of $50 \mu \mathrm{V} \mathrm{s}^{-1}$ is conducted (Fig. 4a). Besides reduction peaks that relates to the transformation of $\mathrm{Li}$ polysulfides to $\mathrm{Li}_{2} \mathrm{~S}_{x}$, there seems other broad current responses emerging when cells are first scanned from 3.0 to $1.5 \mathrm{~V}$. This unusual phenomenon may be greatly associated with undesired electrode polarizations/potential sluggish due to the presence of $\mathrm{NNH}$ outer films that would retard the electrolyte penetration and $\mathrm{Li}^{+}$transfer. In subsequent second and fourth cathodic scans, the intensity of these current signals is gradually diminished. Whereas, in the 10th scan, two well-defined reduction peaks above $\sim 2.0 \mathrm{~V}$ are present, and very little current trace in a potential scope of $\sim 1.64-1.8 \mathrm{~V}$ is noticed. We suppose that these current signals appearing in the low potential range $(<1.85 \mathrm{~V})$ should be linked to electrochemical interactions among the electrolyte, $\mathrm{Li}^{+}$and NNH. To prove this consumption, a CV scan towards the CB@NNH hybrids (without active $S_{8}$ ) under the same electrochemical conditions is performed (Fig. $4 \mathrm{~b}$ ). In the initial CV scan, there emerges a strong irreversible current peak (its intensity is still negligible when compared with that for $\mathrm{S}_{8}$-contained cases) located at the position of $\sim 1.5-1.86 \mathrm{~V}$ whose potential scope is highly in line with our records in Fig. 4a. This result may properly support our above estimation. With the increase of CV scans, the intensity for such current signals tends to decrease gradually and at last almost vanishes over 10 cycles, which implies the termination of these irreversible reactions. This electrochemical process may lead to 
a

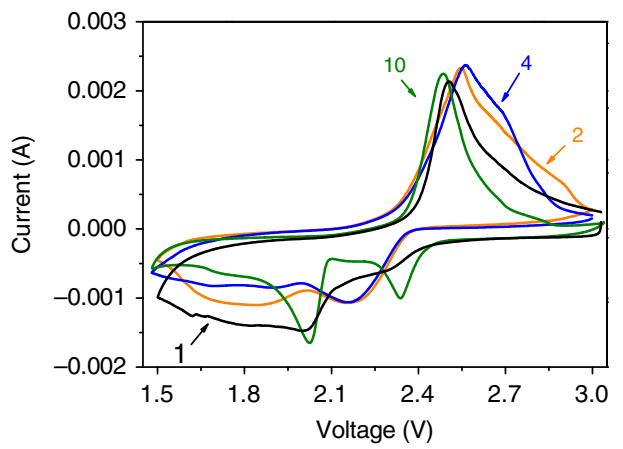

b

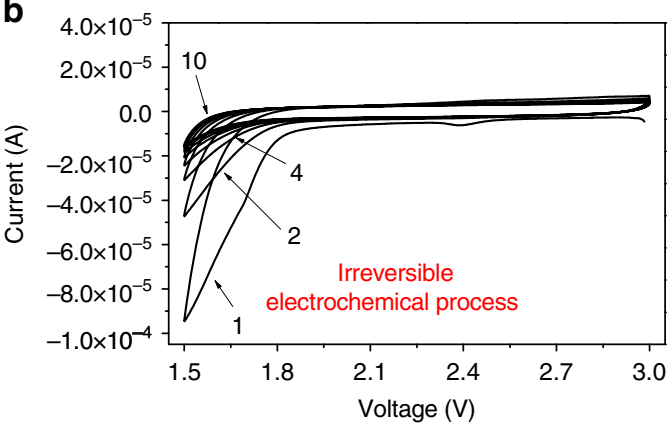

Figure 4 | Electrochemical characterizations of the $\mathbf{S}_{\mathbf{8}} @ \mathbf{C B} @ N N H$

cathode. $C V$ testing of (a) $\mathrm{S}_{8} @ C B @ N N H$ cathode and (b) CB@NNH under a scan rate of $50 \mu \mathrm{Vs}^{-1}$.

the vast generation of complex $\mathrm{Li}$, Ni-mixed hydroxides on $\mathrm{S}_{8} @ \mathrm{CB}$ particle surface.

Deep understanding on the working principles of $\mathrm{NNH}$ in $\mathrm{Li} / \mathrm{S}$ cells is made using ex situ s.e.m. monitoring coupled with precise EDX probing, Raman spectroscopy, XRD and surface-sensitive X-ray photoelectron spectroscopy (XPS) measurements based on the disassembly of cycled cells at the charge-end state of 50th, 300 th and 500th, respectively. Figure 5a-d in turn displays representative s.e.m. images of disassembled cells after different cycles. The top-view s.e.m. image (Fig. 5a) clearly depicts that bulky $\mathrm{S}_{8} @ \mathrm{CB} @ \mathrm{NNH}$ particles remain densely packed and well embedded in the film electrode after 50 times of full charge. In addition, there are layered structures definitely filled in electrode matrices. The electrode suffering from 300 continual cycles looks similar to that in the former case. Though the cathode film to some extent becomes loose possibly due to volume expansions and structural reconfigurations of electrode during lithiation/ delithiation, the close encapsulation of protective armors on $\mathrm{S}_{8} @ \mathrm{CB}$ particles is always maintained (Fig. 5b). Attentions to morphological features of cycled $\mathrm{S}_{8} @ \mathrm{CB} @ \mathrm{NNH}$ have been paid specially. A zoom-in s.e.m. observation on a selected edge place (Fig. 5c) discloses that the subunits of $\mathrm{S}_{8} @ \mathrm{CB} @ N N H$ (size: $50 \sim 150 \mathrm{~nm}$, a bit larger than pristine $\mathrm{S}_{8} @ \mathrm{CB}$ unit) are still underneath the protection of gel-like film structures despite the situation that cells have run uninterruptedly for hundreds of cycles. The geometric observation on the cathode (Fig. 5d) unambiguously demonstrates that bulks of $\mathrm{S}_{8} @ \mathrm{CB} @ \mathrm{NNH}$, though turning into porous structures in whole or in part, are able to survive a long-time cyclic test over 500 times charge and discharge (lasting for more than half a year) and still preserve an integrated electrode construction. Line-scan analysis across one cycled $\mathrm{S}_{8} @ \mathrm{CB} @ \mathrm{NNH}$ particle (Fig. 5e) exhibits a uniform elemental distribution of $\mathrm{C}, \mathrm{S}, \mathrm{O}$ and $\mathrm{Ni}$, proving that the functionalized hybrid configurations cannot be varied tremendously even if suffering from long-term input/output operations.
EDX testing towards different particle zones (Fig. 5f) give out an identical result, reconfirming the good retention ability on electrode constituents. XRD measurement is used to identify the chemical compositions of cycled $\mathrm{S}_{8} @ \mathrm{CB} @ \mathrm{NNH}$ after 50 cycles (Supplementary Fig. 10a). The presence of a broad diffraction signal instead of original sharp peaks illustrates that the primal $\mathrm{S}_{8}$ crystals have evolved into an amorphous state. Moreover, a wide (001) diffraction peak also turns up at $20.4^{\circ}$, which is well indexed to the (001) phase of LiOH (JCPDS no. 32-0564). To illustrate $\mathrm{LiOH}$ comes from reactions between $\mathrm{Li}^{+}$and $\mathrm{NNH}$, Raman spectroscopy is implemented on cycled electrode of $\mathrm{CB} @ \mathrm{NNH}$ purposely. In Raman spectrum (Supplementary Fig. 10b), we readily distinguish the characteristic peaks of $\mathrm{LiOH}$, which are successively assigned to $A_{1 g}$ mode $\left(329 \mathrm{~cm}^{-1}\right)$ and $E_{g}$ mode $\left(531 \mathrm{~cm}^{-1} \text { and } 620 \mathrm{~cm}^{-1}\right)^{37}$, revealing a fact that $\mathrm{LiOH}$ is indeed produced after irreversible electrochemical reactions mentioned above. XPS testing is further used to determine the chemical state of $\mathrm{Ni}$ in the formed composites coating on $\mathrm{S}_{8} @ \mathrm{CB}$. High-resolution Ni 2p XPS spectrum (Fig. $5 \mathrm{~g}$ ) shows that two prominent peaks (indexed to $\mathrm{Ni} 2 \mathrm{p}_{3 / 2}$ and $\mathrm{Ni} 2 \mathrm{p}_{1 / 2}$ ) are located at binding energies of 857.8 and $875.2 \mathrm{eV}$, with satellite peaks at high binding energies of 863.5 and $880.4 \mathrm{eV}$, respectively. All these features have been fully evidenced in the literatures as typical fingerprints of $\mathrm{Ni}(\mathrm{III})$ oxidation state (rather than the initial $\mathrm{Ni}(\mathrm{II})$ state $)^{38}$. The variation in chemical valence hints that the involved $\mathrm{Ni}$ element in armors may also take part in redox reactions, possibly acting as a mediator/catalyst role via interactions with polysulfides to improve the reaction kinetics ${ }^{40}$, and contribute to the cell capacity (thus the total specific capacity is even a bit higher than that of $\left.S_{8}\right)$. To further understand the interactions among pure $\mathrm{NNH}$ (exclude $\mathrm{CB}$ since $\mathrm{CB}$ itself has a reversible capacity when working in cell systems), $\mathrm{Li}^{+}$and polysulfides in Li/S cells, more systematic researches will be highly encouraged and concentrated in our future work via using distinct binders (like CMC, LA132 and so on). The CV comparisons between the $\mathrm{S}_{8} @ \mathrm{CB} @ \mathrm{NNH}$ and $\mathrm{S}_{8} @ \mathrm{CB}$ electrodes after 100 cycles (Supplementary Fig. 11) may grant us important hints to account for the capacity exceeding as well as extended plateaus. Clearly, S8@CB@NNH exhibits a higher current intensity than $\mathrm{S}_{8} @ \mathrm{CB}$ owing to its preferable capacity retention. Notice that except for signal increases at/around peak positions, additional current enhancements are found in other potential regions (for example, from $\sim 2.0$ to $2.3 \mathrm{~V}$ ) as well. This implies that besides electrochemical reactions of $S_{8}$ (the main capacity contributor), other gentle reversible conversions in cathode systems (typically like pseudocapacitive and inner redox reactions or interactions and so on) may give partial contributions to the ultimate cell capacity.

Above characteristics and evidences guide us to unveil the working mechanism of $\mathrm{NNH}$ in $\mathrm{Li} / \mathrm{S}$ cathodes, as schematically described in Fig. 5h. Among primal tens of cycles, the majority of thin-layered NNHs surrounding the $\mathrm{S}_{8} @ \mathrm{CB}$ particles are gradually transformed into more electrochemically stabilized Li-based hydroxide compounds via mild irreversible discharge reactions without causing any structure collapses or film pulverizations. Abundant incorporation of hydrophilic and surface hydroxyl groups that are reported to bind favourably with polysulfide anions ${ }^{12,39}$, combined by a good physicochemical stability of such derived substances could effectively assure the suppression of polysulfides dissolution and leakage. This has been testified by good electrochemical properties of $\mathrm{S}_{8} @ \mathrm{CB} @ N N H$, with remarkable extended cyclability, greatly enhanced $\mathrm{S}_{8}$ utilization and fairly high coulombic efficiencies (all the time retaining $\sim 98 \%$ above). Moreover, the multi-layered armors compactly 'worn' on $\mathrm{S}_{8} @ \mathrm{CB}$ would never deteriorate the ionic transfer property. On one hand, rich $\mathrm{Li}^{+}$in electrolyte 

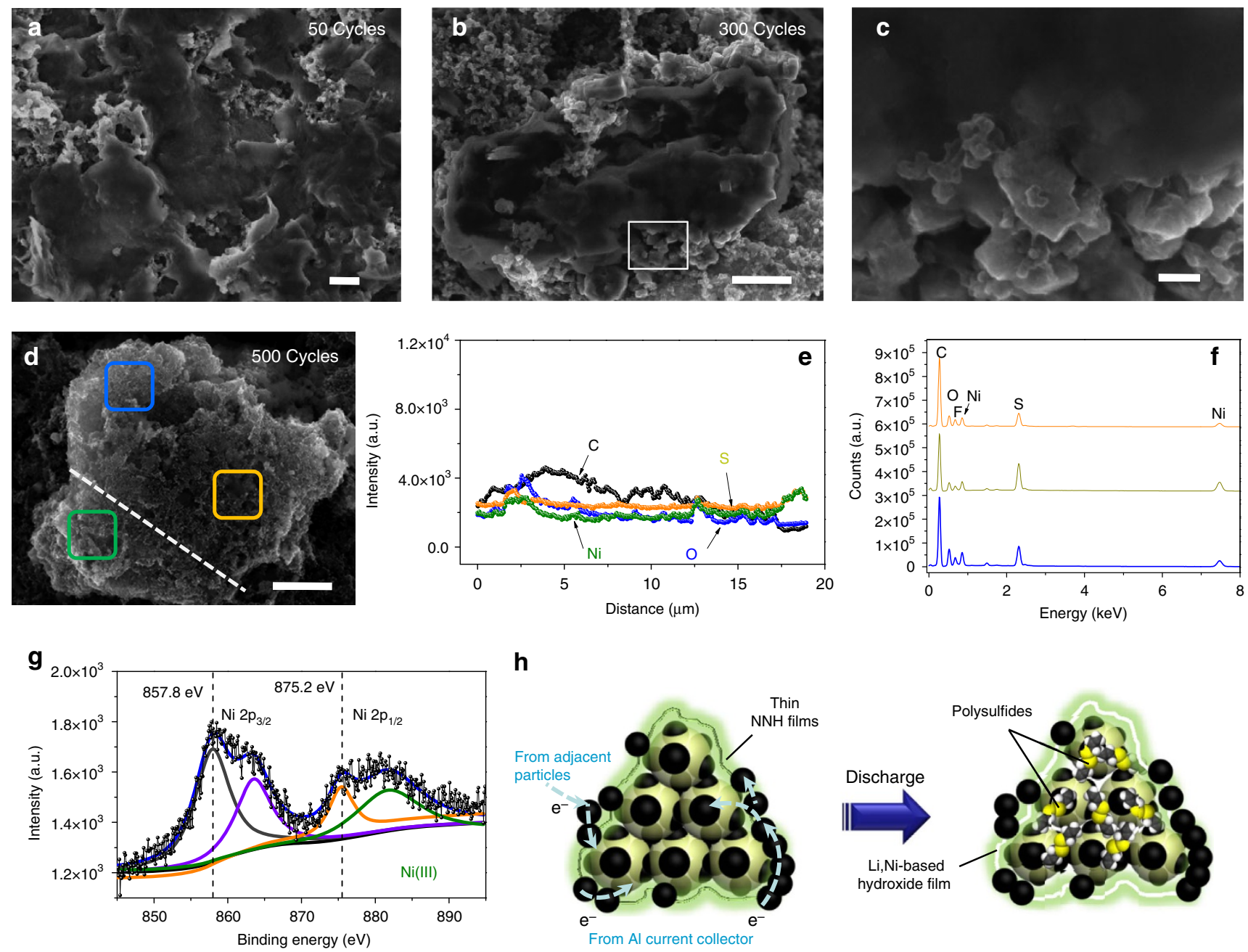

h
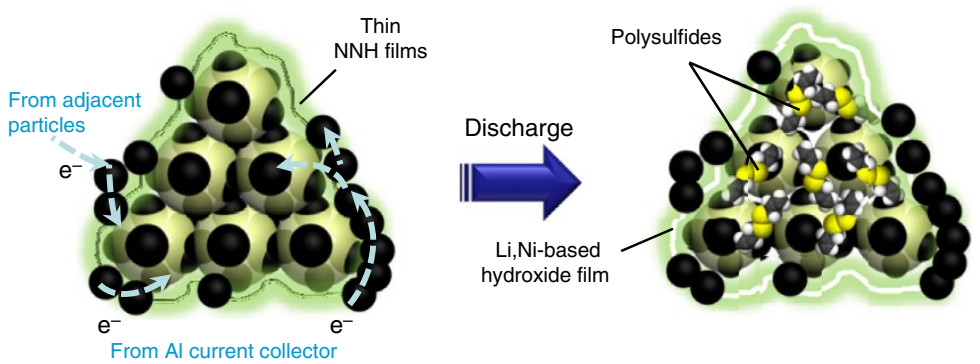

Figure 5 | Characterizations of cycled $\mathbf{S}_{\mathbf{8}} @ \mathbf{C B} @ \mathbf{N N H}$ cathodes. (a-d) The ex situ s.e.m. monitoring the cathodic morphological changes on increased cyclic numbers. EDX probing towards the S8@CB@NNH cathode after 500 cycles: (e) a line-scan analysis and (f) EDX records on different particle zones. (g) High-resolution Ni 2p XPS spectrum and (h) a schematic showing working mechanisms of NNH in Li/S cathodes. Proper pathways for electron transfer in the discharge stage have also been displayed. Scale bars, $10 \mu \mathrm{m}(\mathbf{a}, \mathbf{b}, \mathbf{d})$ and $100 \mathrm{~nm}(\mathbf{c})$.

phase may enter the inner places of $\mathrm{S}_{8} @ \mathrm{CB} @ \mathrm{NNH}$ by means of $\mathrm{Li}^{+}$exchange with $\mathrm{Li}$, Ni-mixed hydroxides wrapping outside the $\mathrm{S}_{8} @ \mathrm{CB}$. On the other hand, the massive and rapid ionic migrations/diffusions can proceed smoothly throughout effortlessly traversing micropores/mesopores that intrinsically exist on $\mathrm{NNH}$ films or in situ created by $\mathrm{Li}^{+}$on thin-layered structures during deep charge/discharge cycling.

\section{Discussion}

In this work, the thin-layered Ni-based hydroxide has been demonstrated for the first time as one feasible and effective encapsulation material to prolong the service life of $\mathrm{Li} / \mathrm{S}$ cells. Our uncustomary selection of hydroxides as an encapsulation candidate is highly triggered by three critical factors as follows:

First and foremost, there exist electrochemically irreversible interactions between $\mathrm{Li}^{+}$and layered hydroxides, leading to the generation of a more stabilized shelly structure. This shelly structure can function as a robust protective layer to entrap the active $S_{8}$ and intermediate polysulfide molecules inside the cathodes, hence efficiently extending the cell cycle lifespan.

Next, our used layered NNH itself is pretty thin. With that, this gauzy covering layer allows the ease of $\mathrm{Li}^{+}$access into the cathode either by diffusions across both interspaces and micropores/mesopores or via $\mathrm{Li}^{+}$exchanges occurring on the solid-liquid interface. This ionic transit is much easier than other cases for thick physical barriers.

Last but never the least, the thin, soft and flexible film prepared in solution phase is apt to worn uniformly on involved precursors no matter what forms and shapes they are present in. This general and simple synthetic strategy, thereby, would pledge the mass production of integrated core-shell hybrid constructions with both intact and intimate wrapping properties.

As a proof-of-concept demonstration, our designed $\mathrm{S}_{8} @ \mathrm{CB} @ \mathrm{NNH}$ hybrid cathode proves great improvements in capacity retention and long-cyclic ability. Unlike previous approaches to trap polysulfides by physical barriers or simple surface interactions, our method is quite efficient, simple, costeffective and particularly suitable for bulky materials, opening up the prospect of using layered thin-metal hydroxides as a creative and effective class of encapsulation materials for promotions of $\mathrm{Li} / \mathrm{S}$ cell performance. Despite these advances, in the way still stand formidable challenges, typically like the presence of undesired potential sluggish/polarizations associated with increased charge-transfer resistances due to tight capsulations of $\mathrm{NNH}$. To decrease such polarizations during the cell operation, further development of better thin shells/layers by means of 
proper and smart engineering/hybridization of $\mathrm{NNH}$ with other robust and conductive substances may hold great promise in future Li/S cell technology and deserve our systematic and elaborate studies.

\section{Methods}

Preparation of $\mathbf{S}_{\mathbf{8}} @ \mathbf{C B}$ hybrid. The $\mathrm{S}_{8} @ \mathrm{CB}$ hybrid was prepared following a melt-diffusion strategy. $S_{8}$ powders (8 g; Sigma-Aldrich, Index-No. 016-094-00-1; assay $\geq 99.5 \%)$ and CB (2 g; LITX 50, Cabot China (Shanghai) Ltd.; $\sim 45-60 \mathrm{~m}^{2} \mathrm{~g}^{-1}$ ) were grounded together (the optimal weight ratio of $\mathrm{S}_{8} / \mathrm{CB}$ was found to be $4: 1$ ), and then heated to $155^{\circ} \mathrm{C}$ and maintained for $6 \mathrm{~h}$.

Preparation of $\mathbf{S}_{\mathbf{8}} @ \mathbf{C B} @ N$ NH hybrid. The $\mathrm{S}_{8} @ \mathrm{CB} @ N N H$ hybrid was synthesized by using $\mathrm{S}_{8} @ \mathrm{CB}$ hybrids as the starting materials. In details, a mixture of $\mathrm{S}_{8} @ \mathrm{CB}$ powders $(0.6 \mathrm{~g})$, hexamethylenetetramine $/ \mathrm{C}_{6} \mathrm{H}_{12} \mathrm{~N}_{4}, 0.5 \mathrm{~g}$ (Sigma-Aldrich, IndexNo. 612-101-00-2; assay $\geq 99.0 \%), \mathrm{Ni}\left(\mathrm{NO}_{3}\right)_{2} \cdot 6 \mathrm{H}_{2} \mathrm{O}$ (0.25 g; Sigma-Aldrich, IndexNo. $028-012-00-1$; assay $\geq 97.0 \%)$ and distilled water $(50 \mathrm{ml})$ was magnetically stirred for $30 \mathrm{~min}$. Afterwards, the resulting suspension was transferred into a sealed container $(80 \mathrm{ml})$ and held at $95^{\circ} \mathrm{C}$ for $6 \mathrm{~h}$. Samples were then collected, washed by distilled water several times and dried at $60^{\circ} \mathrm{C}$ in electronic oven.

Characterization. The powder samples were characterized by field-emission SEM (JSM-6700F, $5.0 \mathrm{kV}$ ) collocated with a high-precision energy dispersive spectroscopy system (Oxford Instruments of X-Max ${ }^{\mathrm{N}}$; its silicon drift detector comes in a wide range of detecting sizes, from $20 \mathrm{~mm}^{2}$ for microanalysis up to an astounding $150 \mathrm{~mm}^{2}$ for advanced nanoanalysis), XRD (Bruker D8 Advance diffractometer with $\mathrm{Cu} \mathrm{K} \alpha$ radiation, $\lambda=0.15418 \mathrm{~nm}$ ) and $\mathrm{N}_{2}$ adsorption/desorption (ASAP2020 volumetric adsorption analyser; Micromeritics, USA). The morphology and crystalline structure of $\mathrm{NNH}$ were further characterized using a TEM/high-resolution TEM (JEM 2100F). XPS spectrum was measured on a Perkin-Elmer model PHI 5600 XPS system with a resolution of $0.3-0.5 \mathrm{eV}$ from a monochromated aluminium anode X-ray source. Electrical properties were recorded by the use of a Keithley 4200 semiconductor characterization system (USA). The Raman spectrum was made by using a WITec CRM200 Raman system with $532 \mathrm{~nm}$ excitation laser.

TG analysis was performed on a SDT600 apparatus under a heating rate of $\sim 5 \mathrm{~K} \mathrm{~min}^{-1}$ in Ar atmosphere. For the acid immersion treatment, $1 \mathrm{~g}$ of fresh $\mathrm{S}_{8} @ \mathrm{CB} @ \mathrm{NNH}$ hybrid powder was put into a $100 \mathrm{ml}$ glass beaker wherein a $60 \mathrm{ml}$ of $3 \mathrm{M}$ hydrochloric acid $(\mathrm{HCl})$ solution was contained. This mixture was then subjected to a $30 \mathrm{~min}$ of mild magnetic stirring (rotating rate: $200 \mathrm{rmin}^{-1}$ ) so as to guarantee the complete removal of NNH. The next sample collection procedure is much crucial to the acid immersion treatment. Powders were carefully collected via a slow vacuum filtration process using a microporous membrane filter (Nylon 66; pore size: $0.22 \mu \mathrm{m}$ ), washed with deionized water several times and dried under vacuum conditions at room temperature. To avoid weight losses, nearly all of the residuals on container are thoroughly collected. The powder mass was then determined by a microbalance with an accuracy of $0.01 \mathrm{mg}$ (A\&D Company N92, Japan). According to mass differences, NNH statistically took up $\sim 8.2 \%$ in S8@CB@NNH hybrids.

Electrochemical measurements. The working electrodes were all prepared by mixing powder samples with polyvinylidene difluoride (Sigma-Aldrich, Product No. 427152) binder and CB (weight ratio: 80:10:10) in $N$-methyl-2-pyrrolidone (Sigma-Aldrich, assay $\geq 99 \%$ ) to form a slurry, which was then pasted onto aluminium foil and dried in an electrical oven. Bare $\mathrm{S}_{8} @ \mathrm{CB}$ and $\mathrm{CB} @ \mathrm{NNH}$ electrodes were also made in the same way for electrochemical comparisons. The 2032type coin cells were then assembled in an Ar-filled glovebox (MBRAUN, UNIlab, Germany) by using Li foil as the counter electrode. The used electrolyte was lithium bis(trifluoromethanesulphonyl)imide $(1 \mathrm{M})$ in 1:1 (v/v) 1,2-dimethoxyethane and 1,3-dioxolane (Fosai New Material Co., Ltd. and Kanagawa Co., Ltd., Japan). $\mathrm{LiNO}_{3}$ salt was added ( $\left.1 \mathrm{wt} \%\right)$ to help passivate the $\mathrm{Li}$ anode surface. The mass of electrode materials was measured on a high-accuracy microbalance. The mass ratio of $\mathrm{S}_{8}$ in synthesized $\mathrm{S}_{8} @ \mathrm{CB} @ \mathrm{NNH}$ hybrid is $\sim 78 \%$, while the ratio value in pasted electrodes is $\sim 62.4 \%$ due to additives of $\mathrm{CB}$ and polyvinylidene difluoride. For one electrode, the typical mass loading was $\sim 1.8-2.5 \mathrm{mg} \mathrm{cm}^{-2}$ unless otherwise stated. The specific capacity values are calculated based on the total mass of electrode materials (excluding CB and polymer binders). In cell testing, the charge/discharge profiles and cyclability data were obtained with a programmable battery cycler (Neware Instruments). The $\mathrm{C}$ rates specified in this case study are based on the mass and theoretical capacity of $\mathrm{S}_{8}\left(1 \mathrm{C}=1,675 \mathrm{~mA} \mathrm{~g}^{-1}\right)$. All cells were aged for $8 \mathrm{~h}$ before the cyclic test.

\section{References}

1. Bruce, P. G., Freunberger, S. A., Hardwick, L. J. \& Tarascon, J.-M. Li-O ${ }_{2}$ and Li-S batteries with high energy storage. Nat. Mater. 11, 19-29 (2012).

2. Manthiram, A., Fu, Y. \& Su, Y.-S. Challenges and prospects of lithium-sulfur batteries. Acc. Chem. Res. 46, 1125-1134 (2013).
3. Yang, Y., Zheng, G. Y. \& Cui, Y. Nanostructured sulfur cathodes. Chem. Soc. Rev. 42, 3018-3032 (2013).

4. Wang, J. L., He, Y. S. \& Yang, J. Sulfur-based composite cathode materials for high-energy rechargeable lithium batteries. Adv. Mater. 27, 569-575 (2015).

5. Wang, J. L. et al. Electrochemical characteristics of sulfur composite cathode materials in rechargeable lithium batteries. J. Power Sources 138, 271 (2004).

6. Xin, S. et al. Smaller sulfur molecules promise better lithium-sulfur batteries. J. Am. Chem. Soc. 134, 18510-18513 (2012).

7. Melot, B. C. \& Tarascon, J. M. Design and preparation of materials for advanced electrochemical storage. Acc. Chem. Res. 46, 1226 (2013).

8. Peled, E. \& Yamin, H. Lithium/sulfur organic battery. Prog. Batteries Sol. Cells 5, 56-58 (1984).

9. Yin, Y. X., Xin, S., Guo, Y. G. \& Wan, L. J. Lithium-sulfur batteries: electrochemistry, materials, and prospects. Angew. Chem. Int. Ed. 52, 13186-13200 (2013)

10. Yang, Y. et al. High-capacity micrometer-sized $\mathrm{Li}_{2} \mathrm{~S}$ particles as cathode materials for advanced rechargeable lithium-ion batteries. J. Am. Chem. Soc. 134, 15387-15394 (2012).

11. Chung, S. H. \& Manthiram, A. Bifunctional separator with a light-weight carbon-coating for dynamically and statically stable lithium-sulfur batteries. Adv. Funct. Mater. 24, 5299-5306 (2014).

12. Ji, X., Evers, S., Black, R. \& Nazar, L. F. Stabilizing lithium-sulphur cathodes using polysulphide reservoirs. Nat. Commun. 2, 325 (2011).

13. Zhou, G. M. et al. A graphene-pure-sulfur sandwich structure for ultrafast, long-life lithium-sulfur batteries. Adv. Mater. 26, 625-631 (2014).

14. Su, Y.-S. \& Manthiram, A. A facile in situ sulfur deposition route to obtain carbon-wrapped sulfur composite cathodes for lithium-sulfur batteries. Electrochim. Acta 77, 272-278 (2012).

15. Zheng, G., Yang, Y., Cha, J. J., Hong, S. S. \& Cui, Y. Hollow carbon nanofiber encapsulated sulfur cathodes for high specific capacity rechargeable lithium batteries. Nano Lett. 11, 4462-4467 (2011).

16. Liang, C. D., Dudney, N. J. \& Howe, J. Y. Hierarchically structured sulfur/ carbon nanocomposite material for high-energy lithium battery. Chem. Mater. 21, 4724-4730 (2009).

17. Guo, J., Xu, Y. \& Wang, C. Sulfur-impregnated disordered carbon nanotubes cathode for lithium-sulfur batteries. Nano Lett. 11, 4288-4294 (2011).

18. Jayaprakash, N., Shen, J., Moganty, S. S., Corona, A. \& Archer, L. A. Porous hollow carbon@sulfur composites for high-power lithium-sulfur batteries. Angew. Chem. Int. Ed. 50, 5904-5908 (2011).

19. Zhang, B., Qin, X., Li, G. R. \& Gao, X. P. Enhancement of long stability of sulfur cathode by encapsulating sulfur into micropores of carbon spheres. Energy Environ. Sci 3, 1531-1537 (2010).

20. Zheng, G. et al. Amphiphilic surface modification of hollow carbon nanofibers for improved cycle life of lithium sulfur batteries. Nano Lett. 13, 1265-1270 (2013).

21. Wang, J. L., Yang, J., Xie, J. Y. \& Xu, N. X. A novel conductive polymer-sulfur composite cathode material for rechargeable lithium batteries. Adv. Mater. 14, 963-965 (2002).

22. Zhou, J. W. et al. Rational design of a metal-organic framework host for sulfur storage in fast, long-cycle Li-S batteries. Energy Environ. Sci 7, 2715-2724 (2014)

23. Xiao, L. et al. A soft approach to encapsulate sulfur: polyaniline nanotubes for lithium-sulfur batteries with long cycle life. Adv. Mater. 24, 1176-1178 (2012)

24. Wu, F. et al. Sulfur/polythiophene with a core/shell structure: synthesis and electrochemical properties of the cathode for rechargeable lithium batteries. J. Phys. Chem. C 115, 6057-6063 (2011).

25. Seh, Z. W. et al. Sulphur- $\mathrm{TiO}_{2}$ yolk-shell nanoarchitecture with internal void space for long-cycle lithium-sulphur batteries. Nat. Commun. 4, 1331 (2013).

26. Lee, K. T., Black, R., Yim, T., Ji, X. L. \& Nazar, L. F. Surface-initiated growth of thin oxide coatings for Li-sulfur battery cathodes. Adv. Energy Mater 2, 1490 (2012).

27. Seh, Z. W. et al. Two-dimensional layered transition metal disulphides for effective encapsulation of high-capacity lithium sulphide cathodes. Nat. Commun. 5, 5017 (2014).

28. Bruce, P. G., Scrosati, B. \& Tarascon, J. M. Nanomaterials for rechargeable lithium batteries. Angew. Chem. Int. Ed. 47, 2930-2946 (2008).

29. Wang, Y. G., Li, H. Q., He, P., Hosono, E. \& Zhou, H. S. Nano active materials for lithium-ion batteries. Nanoscale 2, 1294-1305 (2010).

30. Zhu, J. H. et al. Encapsulation of nanoscale metal oxides into an ultra-thin Ni matrix for superior Li-ion batteries: a versatile strategy. Nanoscale 6, 12990-13000 (2014)

31. Jiang, H., Zhao, T., Li, C. Z. \& Ma, J. Hierarchical self-assembly of ultrathin nickel hydroxide nanoflakes for high-performance supercapacitors. J. Mater. Chem. 21, 3818-3823 (2011).

32. Su, Y. S., Fu, Y. Z., Cochell, T. \& Manthiram, A. A strategic approach to recharging lithium-sulphur batteries for long cycle life. Nat. Commun. 4, 2985 (2013).

33. Zhang, S. S. Liquid electrolyte lithium/sulfur battery: fundamental chemistry, problems, and solutions. J. Power Sources 231, 153-162 (2013). 
34. Zhou, G. M. et al. Fibrous hybrid of graphene and sulfur nanocrystals for highperformance lithium-sulfur batteries. ACS Nano 7, 5367-5375 (2013).

35. Zhou, W. et al. Amylopectin wrapped graphene oxide/sulfur for improved cyclability of lithium-sulfur battery. ACS Nano 7, 8801-8808 (2013).

36. Xu, G. L. et al. Porous graphitic carbon loading ultra high sulfur as highperformance cathode of rechargeable lithium - sulfur batteries. ACS Appl. Mater. Interfaces 5, 10782-10793 (2013).

37. Harbach, F. \& Fischer, F. Raman spectra of lithium hydroxide single crystals. J. Phys. Chem. Solids 36, 60l-603 (1975).

38. Grosvenora, A. P. et al. New interpretations of XPS spectra of nickel metal and oxides. Surf. Sci. 600, 1771-1779 (2006).

39. Evers, S., Yim, T. \& Nazar, L. F. Understanding the nature of absorption/ adsorption in nanoporous polysulfide sorbents for the Li-S battery. J. Phys Chem. C 116, 19653-19658 (2012).

40. Liang, X. et al. A highly efficient polysulfide mediator for lithium-sulfur batteries. Nat. Commun. 6, 5682 (2015).

\section{Acknowledgements}

This work was supported by the A STAR SERC PSF grant 1321202101, Singapore National Research Foundation under NRF-RF award no. NRF-RF2010-07 and MOE Tier 2

MOE2012-T2-2-049. W.H. thanks the support by the Natural Science Foundation of Jiangsu Province (BM2012010), Priority Academic Program Development of Jiangsu Higher Education Institutions (YX03001), Ministry of Education of China (IRT1148), Synergetic Innovation Center for Organic Electronics and Information Displays and the National Natural Science Foundation of China (61136003 and 51173081). We thank Dr Chen Shi and Professor Sum Tze Chien at Nanyang Technological University for XPS testing.

\section{Author contributions}

J.J. and T.Y. conceived and designed the experiments. J.J. and J.Z. carried out the experiment and analysed the data. W.A. and X.W. contributed to the discussion and data analysis. Y.W. performed the Raman detection. C.Z. performed the electrical measurement. J.J., J.Z., T.Y. and W.H. discussed the data and wrote the manuscript.

\section{Additional information}

Supplementary Information accompanies this paper at http://www.nature.com/ naturecommunications

Competing financial interests: The authors declare no competing financial interests.

Reprints and permission information is available online at http://npg.nature.com/ reprintsandpermissions/

How to cite this article: Jiang, J. et al. Encapsulation of sulfur with thin-layered nickel-based hydroxides for long-cyclic lithium-sulfur cells. Nat. Commun. 6:8622 doi: 10.1038/ncomms9622 (2015).

(c) (i) This work is licensed under a Creative Commons Attribution 4.0 International License. The images or other third party material in this article are included in the article's Creative Commons license, unless indicated otherwise in the credit line; if the material is not included under the Creative Commons license, users will need to obtain permission from the license holder to reproduce the material. To view a copy of this license, visit http://creativecommons.org/licenses/by/4.0/ 